\title{
Nanocrystals for Electronic and Optoelectronic Applications
}

\author{
Ting Zhu, ${ }^{1}$ Sylvain G. Cloutier, ${ }^{2}$ Ilia Ivanov, ${ }^{3}$ Kenneth L. Knappenberger Jr., \\ Istvan Robel, ${ }^{5}$ and Fan Zhang ${ }^{6}$
}

${ }^{1}$ Philips Lumileds, San Jose, CA 95131, USA

${ }^{2}$ University of Delaware, Newark, DE 19716, USA

${ }^{3}$ Oak Ridge National Lab, Oak Ridge, TN 37831, USA

${ }^{4}$ Florida State University, Tallahassee, FL 32306, USA

${ }^{5}$ Los Alamos National Laboratory, Los Alamos, NM 87545, USA

${ }^{6}$ Cree Inc., Santa Babara, CA 93117, USA

Correspondence should be addressed to Ting Zhu, txz902@gmail.com

Received 23 January 2012; Accepted 23 January 2012

Copyright (C) 2012 Ting Zhu et al. This is an open access article distributed under the Creative Commons Attribution License, which permits unrestricted use, distribution, and reproduction in any medium, provided the original work is properly cited.

Electronic and optoelectronic devices, from computers and smart cell phones to solar cells, have become a part of our life. Currently, devices with featured circuits of $45 \mathrm{~nm}$ in size can be fabricated for commercial use. However, further development based on traditional semiconductor is hindered by the increasing thermal issues and the manufacturing cost. During the last decade, nanocrystals have been widely adopted in various electronic and optoelectronic applications. They provide alternative options in terms of ease of processing, low cost, better flexibility, and superior electronic/optoelectronic properties. By taking advantage of solution-processing, self-assembly, and surface engineering, nanocrystals could serve as new building blocks for low-cost manufacturing of flexible and large area devices. Tunable electronic structures combined with small exciton binding energy, high luminescence efficiency, and low thermal conductivity make nanocrystals extremely attractive for FET, memory device, solar cell, solid-state lighting/display, photodetector, and lasing applications. Efforts to harness the nanocrystal quantum tunability have led to the successful demonstration of many prototype devices, raising the public awareness to the wide range of solutions that nanotechnology can provide for an efficient energy economy. This special issue aims to provide the readers with the latest achievements of nanocrystals in electronic and optoelectronic applications, including the synthesis and engineering of nanocrystals towards the applications and the corresponding device fabrication, characterization and computer modeling.

This special issue contains eight papers, in which four papers discuss the nanocrystal applications in electronic and optoelectronic devices. They are followed by three papers regarding the synthesis and characterization of the optical and optoelectronic properties of nanomaterials. The last one introduces an interesting application of nanoparticles in food science benefiting from their optical properties.

In the paper, "Facile synthesis of colloidal $\mathrm{CuO}$ nanocrystals for light-harvesting applications", Y.-F. Lim et al. present a synthesis of $\mathrm{CuO}$ nano-crystals by a facile alcohothermal route, which enables the processing of these materials by solution. They also demonstrate a bilayer solar cell comprising of $\mathrm{CuO}$ nanocrystals and phenyl-C61-butyric acid methyl ester (PCBM) with a power conversion efficiency of $0.04 \%$, indicating the potential of this material for light-harvesting applications.

In the paper entitled "Developing quantum dot phosphorbased light emitting diodes for aviation lighting applications," J.-M. Wu et al. investigated the feasibility to use quantum dot (QD) phosphor-based LED in night vision imaging systems for aviation applications. In their proof-of-concept experiment, they have demonstrated the great potential of QD phosphor-based LEDs in cockpit illumination and back light source of monitor screens, as well as the LED indicator lights of aviation panels.

In the paper entitled "Temperature dependence of electrical characteristics of carbon nanotube field-effect transistors: a quantum simulation study," S. M. Noorhakhsh et al. investigate the attributes of carbon nanotube field-effect transistors (CNTFET) in different temperatures by a 2-dimensional full quantum simulation. It has shown that temperature increase results in higher subthreshold swing and low 
on-off current ratio. The results are considered to be useful for design considerations in those devices.

In the paper entitled "Employing photo-assisted ligand exchange technique in layered quantum dot LEDs," W. Hu et al. present a photo-assisted ligand exchange approach to replace the oleic acid ligand molecules over PbSe quantum dots (QDs). The surface-treated QDs are used to fabricate thin-film QD-LEDs, which results in improved LED performance over the untreated QD-LEDs.

In the paper entitled "Synthesis of $\mathrm{SnO}_{2}-\mathrm{ZnO}$ core-shell nanowires and their optoelectronic properties," K.-Y. Pan et al. present a method of synthesizing core/shell semiconductor nanowires using a step-by-step atomic layer deposition process. The hybrid $\mathrm{SnO}_{2}-\mathrm{ZnO}$ nanowires have significantly improved conductance and faster photoresponse compared to pure $\mathrm{SnO}_{2}$ nanowires, making them more suitable for optoelectronic applications.

In the paper entitled "Fluorescent properties of $\mathrm{ZnO}$ nanostructures fabricated by hydro-thermal method," Z. Dong et al. synthesized $\mathrm{ZnO}$ nanorods with mean diameter of $200 \mathrm{~nm}$ on different substrates by hydrothermal method. They investigated the nanorod fluorescent properties by linear and nonlinear excitation as well as Raman spectrum.

In the paper entitled "Study of structural and optical properties of zinc oxide rods grown on glasses by chemical spray pyrolysis," E. Sonmez et al. investigate morphological and optical properties of zinc oxide rods prepared by spray pyrolysis. The results indicate that spray pyrolysis is a cheap and viable technique for producing high quality $\mathrm{ZnO}$ rods for optical applications.

In the final paper "Gold nanoparticle sensor for the visual detection of pork adulteration in meatball formulation," M. E. Ali et al. use gold nanoparticles as colorimetric sensor to detect pork adulteration in beef/chicken preparations. The method is cheap, reliable, and simple, depending on the color change of $20 \mathrm{~nm}$ gold nanoparticles following salt addition. The result can be further confirmed by the absorption spectroscopy. The $\mathrm{A}_{1 \mathrm{~L}}$ optical mode was found important to the fluorescent properties of $\mathrm{ZnO}$ materials.

Ting Zhu

Sylvain G. Cloutier

Ilia Ivanov

Kenneth L. Knappenberger Jr.

Istvan Robel

Fan Zhang 

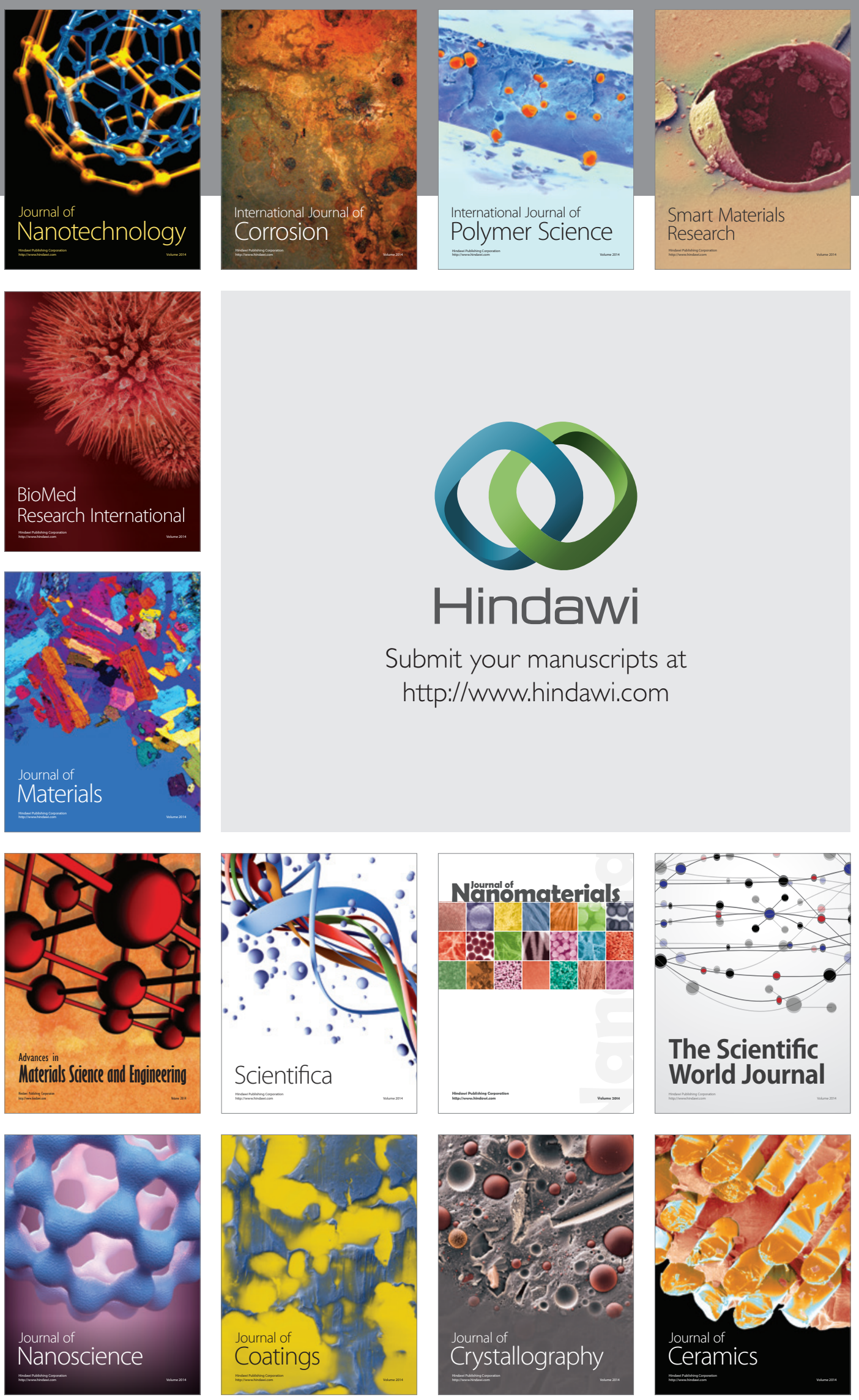

The Scientific World Journal

Submit your manuscripts at

http://www.hindawi.com

\section{World Journal}

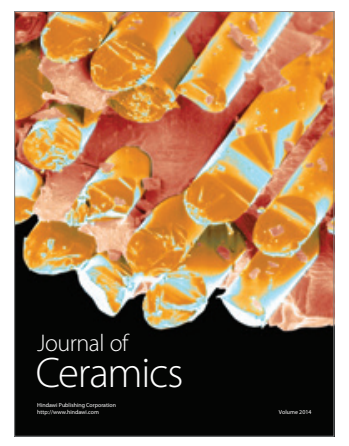

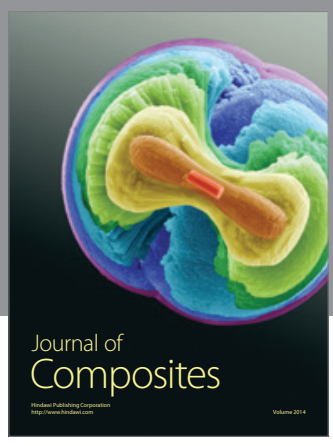
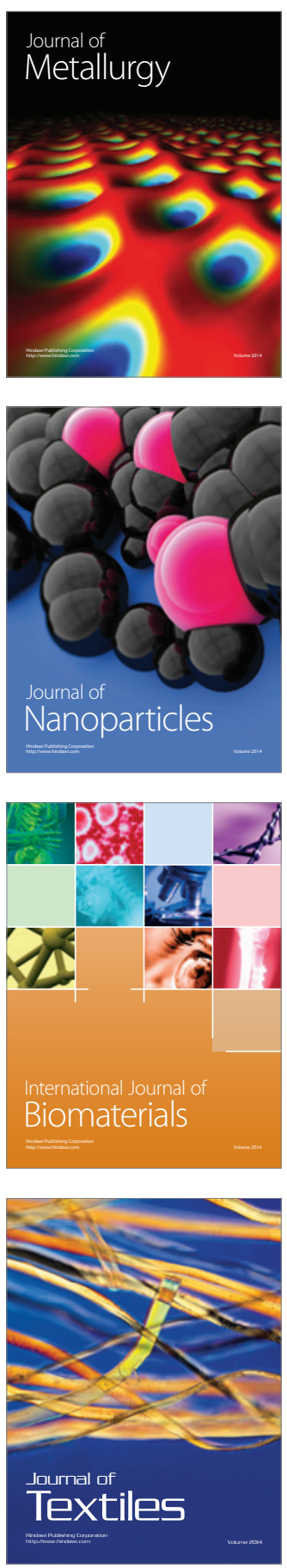